\title{
Longitudinal prediction of outcome in idiopathic pulmonary fibrosis using automated CT analysis
}

\author{
To the Editor:
}

The advent of antifibrotic agents $[1,2]$ as standard of care in idiopathic pulmonary fibrosis (IPF) requires that new non-inferiority IPF drug trials will need to identify smaller declines of forced vital capacity (FVC). Marginal annualised FVC declines (between 5.00 and 9.99\%) are particularly challenging to interpret as they might reflect measurement variation or genuine clinical deterioration [3]. Following on from previous baseline-only computed tomography (CT) analyses [4], the current study examined whether changes in computer features (CALIPER) across serial CT examinations could be considered as a trial co-endpoint, particularly with regard to adjudicating marginal FVC declines, and therefore improve the sensitivity of IPF drug trials.

Previous baseline IPF analyses identified that variable initiation time, dosages, durations and types of antifibrotic medication in study participants had a profound confounding effect on mortality relationships [4]. Consequently, analyses in the current manuscript were restricted to IPF patients not receiving anti-fibrotic therapy (discovery cohort: $n=71$ Royal Brompton Hospital patients presenting from January 2007 to December 2014); validation cohort: $n=24$ St Antonius Hospital, Nieuwegein patients presenting from January 2005 to June 2014 and n=23 Mayo Clinic Rochester patients presenting from January 2009 to June 2015). All patients had two non-contrast volumetric CT scans between 5 and 30 months apart (mean CT interval: discovery cohort 1.1 years; validation cohort 1.2 years) as part of their clinical care. Baseline diffusion capacity of the lung for carbon monoxide $\left(D_{\mathrm{LCO}}\right)$ and FVC (baseline and longitudinal) were collected if performed within 3 months of the respective CTs. No patients were lost to follow-up.

Annualised FVC change was measured using a linear mixed effects model on all eligible timepoints to derive the best linear unbiased predictor (BLUP) as previously described using the lmer function from the $\mathrm{R}$ package lme4 [5]. A naïve estimate of FVC change was also examined using FVC measurements at the first and second CT timepoint. For the naïve estimate we computed annual relative change by dividing the absolute annual change by the baseline FVC value (relative). Dichotomised relative FVC declines $(\geqslant 5 \%$ or $\geqslant 10 \%$ ) were derived based on the naïve and BLUP estimates. Of the 27 CALIPER features examined [4], nine were measured on a whole lung level: total lung volume, normal parenchyma, vessel-related structures (CAL VRS), emphysema, honeycombing, reticular pattern and ground-glass opacity. Fibrosis extent summed reticular pattern and honeycombing. Interstitial lung disease extent additionally summed ground-glass opacification. 18 CAL VRS subdivisions were evaluated, separated according to lung zonal location: upper (UZ), middle (MZ) and lower zones (LZ), and cross-sectional area of structures in each zone: $<5 \mathrm{~mm}^{-2}, 5-10 \mathrm{~mm}^{-2}, 10-15 \mathrm{~mm}^{-2}, 15-20 \mathrm{~mm}^{-2},>20 \mathrm{~mm}^{-2}$. Volumes for all CALIPER features were converted into a percentage using CALIPER-derived total lung volume measurements [6, 7]. Absolute change in the derived 27 CT variables was annualised by dividing by the time interval between the two measurements (in years). Cox proportional hazards models examined CALIPER and FVC change variables in separate discovery and validation cohorts. Time was measured from the second CT. An event was either death $(n=90)$ or transplantation $(n=8)$. Each predictor variable was tested alone while correcting for patient age (at the second CT) and gender. Model fit was evaluated using the concordance index, which assesses how well the ordering of subjects for the actual time of the event agrees with the predicted

@ERSpublications

Change in the vessel-related structures, a computer-derived CT variable, is a strong predictor of outcome in idiopathic pulmonary fibrosis and can increase power in future drug trials when used as a co-endpoint alongside forced vital capacity change http://bit.ly/2M7DfKS

Cite this article as: Jacob J, Bartholmai BJ, van Moorsel CHM, et al. Longitudinal prediction of outcome in idiopathic pulmonary fibrosis using automated CT analysis. Eur Respir J 2019; 54: 1802341 [https://doi. org/10.1183/13993003.02341-2018]. 
time of the event. That is, for all subject pairs it checks whether the subject who had the event first was also the subject predicted to have the event first. A C-index of 0.5 indicates random performance, where in $50 \%$ of cases the subject with the earlier event was predicted to be the subject with the later event. Approval for this study of clinically indicated CT and pulmonary function data was obtained from Liverpool Research Ethics Committee (reference: 14/NW/0028) and the Institutional Ethics Committee of the Royal Brompton Hospital, Mayo Clinic Rochester and St Antonius Hospital, Nieuwegein. Informed patient consent was not required.

Our study findings identified absolute CAL VRS and UZ VRS increases as the strongest survival predictors in discovery and validation cohorts (figure 1a). Both variables were at least equivalent to FVC change when evaluated using C-indices. Significant but weak correlations $\left(r=-0.42, p=1.8 \times 10^{-6}\right)$ were identified between FVC change and absolute VRS change (Pearson's correlation).

Both study populations were then combined and Cox proportional hazards models examined thresholds of CAL VRS and UZ VRS change measured against relative FVC decline thresholds of $\geqslant 5 \%$ and $\geqslant 10 \%$ adjusted for patient age and gender. The predictive performance of FVC-based indicator variables was compared to VRS indicator variables, either used alone or when combined with an FVC-based indicator variable as a "joint endpoint". The joint endpoint reflected whether the FVC decline or the VRS increase was achieved with estimates based on 500 bootstrap replicates $(n=118)$. We estimated the number of additional patients that would reach either $\mathrm{a} \geqslant 5 \%$ or $\geqslant 10 \%$ predicted FVC threshold or a preselected CAL VRS/UZ VRS change threshold in a drug trial setting. Further, we computed the Kaplan-Meier estimator for different subgroups of patients $(n=118)$ depending on whether they reached the FVC or VRS threshold or both (using the SPSS Kaplan-Meier function [8]). Finally, we examined mortality prediction in patients with a BLUP estimated relative decline in FVC of $>5 \%$ but $<10 \%$ not receiving antifibrotics $(n=41)$.

In the combined study population, multivariate Cox mortality models demonstrated that a CAL VRS increase of $\geqslant 0.30 \%$ independently predicted mortality when evaluated against a $\geqslant 10 \%$ FVC decline threshold. When CAL VRS increased by $\geqslant 0.50 \%$, a $\geqslant 10 \%$ FVC decline threshold no longer significantly contributed to mortality prediction. At CAL VRS (figure $1 \mathrm{~b}$ ) and UZ VRS increases of $\geqslant 0.40 \%$, no difference in model C-index was seen when compared to a $\geqslant 10 \%$ FVC decline threshold. The C-index was unchanged when using either a solitary CALIPER endpoint (CAL VRS or UZ VRS increase), or a combined endpoint of an increase in a CALIPER variable and an FVC $\geqslant 10 \%$ decline threshold. Results were maintained when CALIPER variable change thresholds were compared to a $\geqslant 5 \%$ FVC decline threshold.

$79 / 118(67 \%)$ patients reached a CAL VRS of $\geqslant 0.40 \%$ change whilst 54/118 (46\%) reached a $\geqslant 10 \%$ FVC decline threshold ( $\mathrm{p}=0.0003$ ). 89/118 (75\%) patients reached either the CAL VRS threshold of $\geqslant 0.40 \%$ change or $\geqslant 10 \%$ FVC decline threshold (figure 1c). Use of a CAL VRS increase threshold of $\geqslant 0.40 \%$ change identified 35/118 (30\%) more patients reaching an endpoint than the $\geqslant 10 \%$ FVC decline threshold alone. Similarly, at least 30\% more patients reached an endpoint when an UZ VRS threshold was used alongside $\mathrm{a} \geqslant 10 \%$ FVC decline threshold (figure 1c). When CAL VRS and UZ VRS elevation thresholds were examined against $\mathrm{a} \geqslant 5 \%$ FVC decline threshold, additional patients reaching an endpoint were again identified. When all patients with an FVC decline more than $5 \%$ and less than $10 \%$ were subanalysed, CAL VRS thresholds $\geqslant 0.40 \%$ change demonstrated C-indices that were at least equivalent to a $\geqslant 10 \%$ FVC decline threshold (figure 1d).

Our findings demonstrate that in independent discovery and validation populations, an absolute increase in a computer-derived variable, the vessel-related structures (CAL VRS), strongly predicts mortality in IPF patients not exposed to antifibrotic medication. Patients exhibiting a CAL VRS increase $\geqslant 0.40 \%$ were different to those experiencing an FVC decline $\geqslant 10 \%$. Accordingly, if a composite endpoint of CAL VRS $\geqslant 0.40 \%$ increase and/or $\geqslant 10 \%$ FVC decline were used in a drug trial setting, $30 \%$ more patients would reach the composite endpoint than a solitary endpoint of $\geqslant 10 \%$ FVC decline. Our findings also suggest the utility of a CAL VRS threshold $\geqslant 0.40 \%$ increase as an arbitration tool for marginal FVC declines (between 5.0 and $9.9 \%$ ).

The weak correlations between FVC change and VRS change indicate that both variables represent important yet distinct surrogate measures of mortality and argues for their integration as co-endpoints rather than selecting one over another. $A \geqslant 0.40 \%$ increase in VRS across a cohort appeared to be the most accurate measure of change in VRS, when considering both its prognostic effect when judged against FVC decline and its sensitivity as an endpoint. In an individual, whilst the most accurate threshold for VRS change may also be $\mathrm{a} \geqslant 0.40 \%$ threshold, further work is necessary to establish optimal thresholds for use in clinical practice, as just having knowledge of the range of change of a variable does not of course provide any statement of the clinical significance of that change. For example, it was noticeable that more extreme VRS cut-offs, e.g. $0.75 \%$, made even more of a difference in model fit and C-index than a $\geqslant 0.40 \%$ 

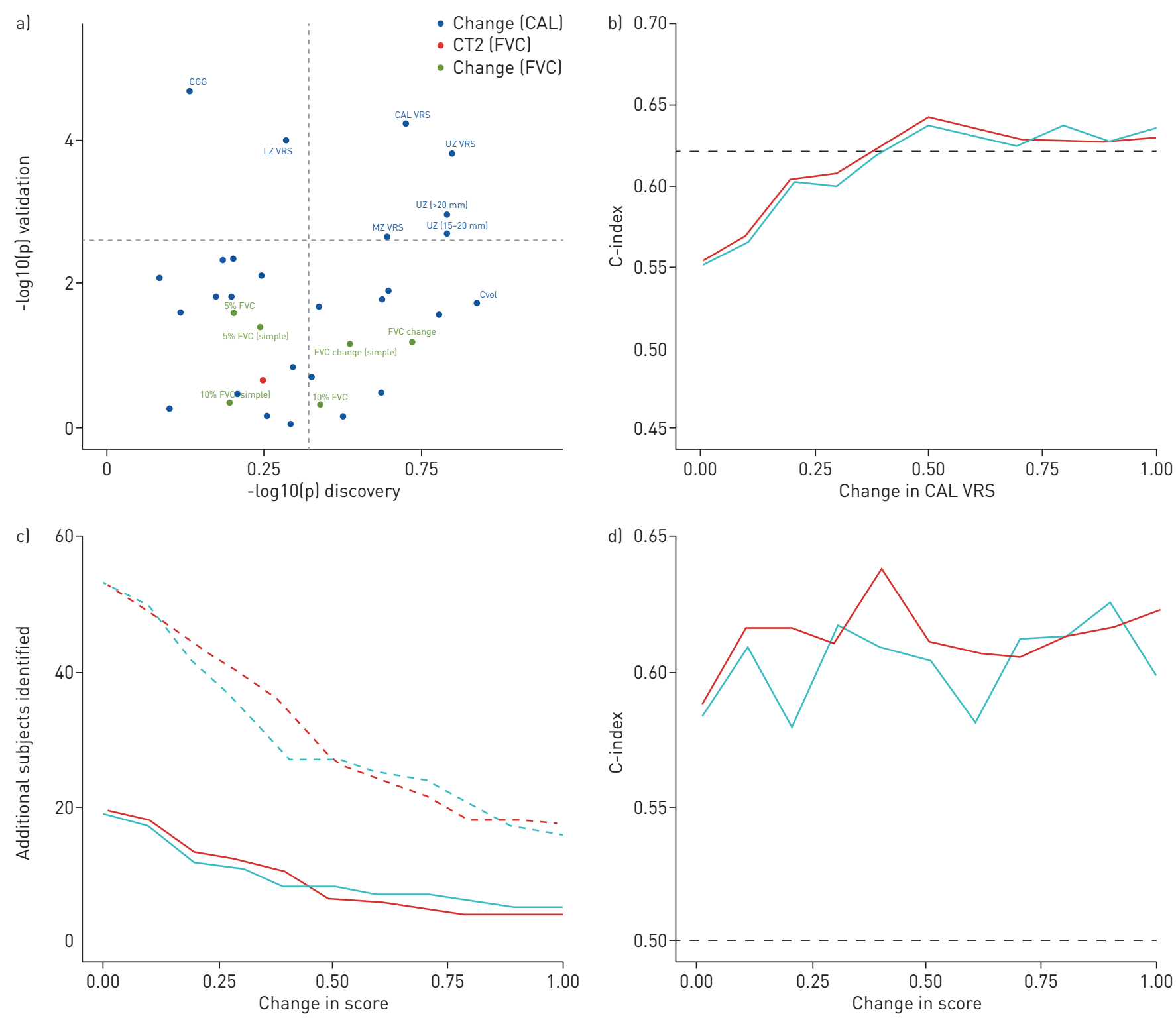

FIGURE 1 a) Scatterplot of - $\log 10$ p-values for various computer-derived (CALIPER) variables (blue points) and forced vital capacity (FVC) decline (green points) in patients not exposed to antifibrotic medication in the discovery cohort ( $x$-axis, $n=71)$ and validation cohort ( $y$-axis, $n=47)$. Horizontal and vertical dotted lines represent the Li and Ji corrected cut-off for statistical significance. FVC decline was calculated using two methods: naïve estimate from two timepoints aligned with the two computed tomography (CT) timepoints (simple) and using best linear unbiased predictions. FVC change was expressed as a continuous variable (FVC change), and at $\geqslant 5 \%$ decline and $\geqslant 10 \%$ decline thresholds. The FVC value at the timepoint of the second CT scan (red dot) was used to benchmark expressions of FVC decline. The pulmonary vessel-related structure score (CAL VRS) was subdivided according to zonal location (UZ VRS: upper zone; MZ VRS: middle zone; LZ VRS: lower zone) and structure cross-sectional area in each zone $\left(<5 \mathrm{~mm}^{-2}, 5-10 \mathrm{~mm}^{-2}, 10-15 \mathrm{~mm}^{-2}, 15-20 \mathrm{~mm}^{-2},>20 \mathrm{~mm}^{-2}\right.$ ). b) C-indices (a measure of goodness of fit of a model) for models examining thresholds of change in CAL VRS examined against a $10 \%$ FVC decline threshold. The horizontal dotted black line indicates the C-index for a 10\% FVC decline threshold model examining the relevant FVC threshold alone. The blue line demonstrates the $\mathrm{C}$-indices for models when a CAL VRS threshold alone was examined. The red line demonstrates the C-indices for models where a binary variable indicated a "joint endpoint", i.e. either the CALIPER or FVC threshold had been reached. c) Additional patients that would reach an endpoint ( $y$-axis), if CAL VRS (red) or upper-zone vessel related structure (UZ VRS, blue) thresholds of change ( $x$-axis) were examined in addition to FVC decline thresholds. The FVC decline thresholds examined included a $\geqslant 5 \%$ FVC decline threshold (solid line) and a $\geqslant 10 \%$ FVC decline threshold (dotted line). d) C-indices (y-axis) for models containing varying thresholds ( $x$-axis) of CAL VRS (red) or UZ VRS (blue) in patients with an FVC between $5 \%$ and $10 \%$. The horizontal dashed black line indicates the $\mathrm{C}$-index 0.5 , i.e. random performance.

threshold. But we cannot know how often such a magnitude of VRS change would be seen in a clinical trial population. A logical next analytic step would therefore be to evaluate VRS change in a well-controlled drug trial population receiving antifibrotics at a standardised dosing regimen.

The validity of VRS change was considered according to the OMERACT filter criteria for IPF clinical trial domains [9]. Regarding truth and discrimination criteria, VRS change was considered to be more 
discriminatory than FVC change at predicting outcome, with potential for use as a continuous variable (with no loss of signal strength), or as a binary threshold alongside an FVC decline threshold to improve endpoint sensitivity. The variable therefore satisfies construct, content and criterion validity and demonstrates sensitivity to change.

The specific impact on VRS change of differing inspiratory effort, acquisition or reconstruction parameters has not been systematically investigated, and further study is indicated. However, our analysis of this measure in a heterogeneous dataset from multiple institutions suggests this is robust. CALIPER outputs are eminently interpretable and feasible to perform but real-world utility of VRS for clinical trials relies on availability of repeated CTs and the computer algorithm, and is therefore limited when compared to FVC measurements.

There were limitations to the current study. Though there were similar average CT intervals between the two study cohorts and change in CT variables were reported as annualised change, the CTs time intervals were not standardised in this retrospective analysis. This lack of standardisation reflects real world clinical practice but may have biased our findings in patients with shorter or longer CT follow up intervals. Whilst the ideal study would have rigorous protocol-led control of serial CT and functional measurements and antifibrotic use, no such study yet exists and were it to begin today, outcome data may only be available several years hence. Accordingly, we believe our analyses capture a realistic contemporary cross-section of IPF data points.

In conclusion, we have demonstrated for the first time that change in a computer-derived variable, vessel-related structures, which has no visual correlate is a powerful surrogate for mortality in IPF. VRS change correlates weakly with FVC change and identifies different poor-outcome patients than a $\geqslant 10 \%$ FVC decline threshold. Use of a VRS threshold of $\geqslant 0.40 \%$ change alongside a $\geqslant 10 \%$ FVC decline threshold can identify $30 \%$ more patients that reach an endpoint and argues for the consideration of VRS change as an IPF drug trial co-endpoint to adjudicate indeterminate FVC declines of 5.0-9.9\%.

Joseph Jacob $\oplus^{1,2}$, Brian J. Bartholmai ${ }^{3}$, Coline H.M. van Moorsel ${ }^{4,5}$, Srinivasan Rajagopalan ${ }^{3}$, Anand Devaraj, Hendrik W. van $\mathrm{Es}^{7}$, Teng Moua ${ }^{8}$, Frouke T. van Beek ${ }^{4}$, Ryan Clay ${ }^{8}$, Marcel Veltkamp ${ }^{4,5}$, Maria Kokosi ${ }^{9}$, Angelo de Lauretis $^{10}$, Eoin P. Judge ${ }^{11}$, Teresa M. Jacob ${ }^{12}$, Tobias Peikert ${ }^{8}$, Ronald Karwoski ${ }^{13}$, Fabien Maldonado ${ }^{14}$, Elisabetta Renzoni ${ }^{9}$, Toby M. Maher ${ }^{15,16}$, Andre Altmann $\oplus^{2}$ and Athol U. Wells ${ }^{9}$

${ }^{1}$ Dept of Respiratory Medicine, University College London, London, UK. ${ }^{2}$ Centre for Medical Image Computing, University College London, London, UK. ${ }^{3}$ Dept of Radiology, Mayo Clinic Rochester, Rochester, MN, USA. ${ }^{4}$ St Antonius ILD Center of Excellence, Dept of Pulmonology, St. Antonius Hospital, Nieuwegein, The Netherlands. ${ }^{5}$ Division of Heart and Lungs, University Medical Center Utrecht, Utrecht, The Netherlands. ${ }^{6}$ Dept of Radiology, Royal Brompton Hospital, London, UK. ${ }^{7}$ Dept of Radiology, St. Antonius Hospital, Nieuwegein, The Netherlands. ${ }^{8}$ Dept of Pulmonary Medicine, Mayo Clinic Rochester, Rochester, MN, USA. ${ }^{9}$ Interstitial Lung Disease Unit, Royal Brompton Hospital, Royal Brompton and Harefield NHS Foundation Trust, London, UK. ${ }^{10}$ Division of Pneumology, "Guido Salvini" Hospital, Garbagnate Milanese, Italy. ${ }^{11}$ Dept of Respiratory Medicine, Aintree University Hospital, Liverpool, UK. ${ }^{12}$ Dept of Radiology, St Georges Hospital, London, UK. ${ }^{13}$ Dept of Physiology and Biomedical Engineering, Mayo Clinic Rochester, Rochester, MN, USA. ${ }^{14}$ Division of Allergy, Pulmonary and Critical Care Medicine, Vanderbilt University Medical Center, Nashville, TN, USA. ${ }^{15}$ NIHR Respiratory Clinical Research Facility, Royal Brompton Hospital, London, UK. ${ }^{16}$ Fibrosis Research Group, National Heart and Lung Institute, Imperial College, London, UK.

Correspondence: Joseph Jacob, Dept of Respiratory Medicine, Rayne Institute, University Street, University College London, WC1E 6JF, UK. E-mail: j.jacob@ucl.ac.uk

Received: 13 Sept 2018 | Accepted after revision: 15 May 2019

Author contributions: J. Jacob, A. Altmann, F.T. van Beek, C.H.M. van Moorsel, M. Veltkamp, H.W. van Es, R. Clay, T.M. Jacob, T. Moua, E.P. Judge, A. de Lauretis, A. Devaraj, T. Peikert, F. Maldonado, M. Kokosi, T.M. Maher, E. Renzoni and A.U. Wells were involved in either the acquisition, or analysis or interpretation of data for the study. J. Jacob and A.U. Wells were also involved in the conception and design of the study. B.J. Bartholmai, R. Karwoski and S. Rajagopalan invented and developed CALIPER. They were involved in processing the raw CT scans and in generation of figures but were not involved with the analysis or interpretation of the data in the study. All authors revised the work for important intellectual content and gave final approval for the version to be published. J. Jacob agrees to be accountable for the all aspects of the work in ensuring that questions related to the accuracy or integrity of any part of the work are appropriately investigated and resolved.

Conflict of interest: J. Jacob reports personal fees for advisory board work from Boehringer Ingelheim, outside the submitted work. B.J. Bartholmai reports grants (paid to Mayo Clinic) from Royal Brompton Hospital, personal fees from Promedior and Boehringer Ingelheim, during the conduct of the study; royalties (paid to Mayo Clinic) from Imbio, LLC, outside the submitted work; and has a patent Systems and Methods for Analysing in vivo Tissue Volumes using Medical Imaging Data licensed to Imbio LLC. C.H.M. van Moorsel has nothing to disclose. S. Rajagopalan reports grants (paid to Mayo Clinic) from Royal Brompton Hospital, during the conduct of the study; royalties (paid to Mayo Clinic) from Imbio, LLC, outside the submitted work; and has a patent Systems and Methods for Analysing in vivo Tissue Volumes using Medical Imaging Data licensed to Imbio LLC. A. Devaraj reports personal fees from Boehringer Ingelheim and Roche, outside the submitted work. H.W. van Es has nothing to disclose. T. Moua has nothing to disclose. F.T. van Beek has nothing to disclose. R. Clay has nothing to disclose. M. Veltkamp has nothing to disclose. 
M. Kokosi has nothing to disclose. A. de Lauretis has nothing to disclose. E.P. Judge has nothing to disclose. T.M. Jacob has nothing to disclose. T. Peikert has nothing to disclose. R. Karwoski reports grants (paid to Mayo Clinic) from Royal Brompton Hospital, during the conduct of the study; royalties (paid to Mayo Clinic) from Imbio, LLC, outside the submitted work; and has a patent Systems and Methods for Analysing in vivo Tissue Volumes using Medical Imaging Data licensed to Imbio LLC. F. Maldonado has nothing to disclose. E. Renzoni reports personal fees for lectures from Roche and Takeda, personal fees for lectures and advisory board meetings from Boehringher, outside the submitted work. T.M. Maher is an investigator in an ongoing Phase $2 \mathrm{~b}$ study for Gilead; reports grants and personal fees for advisory board work from GSK, grants from Novartis, personal fees from Boehringer Ingelheim InterMune, Lanthio, Sanofi Aventis, AstraZeneca, Roche, Bayer, Biogen Idec, Cipla, Prometic and Apellis, grants, personal fees and research fees (paid to institution) from UCB, outside the submitted work. A. Altmann has nothing to disclose. A.U. Wells reports personal fees for lectures and advisory board work from Intermune, Boehringer Ingelheim, Roche and Bayer, personal fees for advisory board work from Gilead and MSD, personal fees for lectures from Chiesi, outside the submitted work.

Support statement: A. Altmann holds an MRC eMedLab Medical Bioinformatics Career Development Fellowship. This work was supported by the Medical Research Council (grant number MR/L016311/1), the National Institute of Health Research Respiratory Disease Biomedical Research Unit at the Royal Brompton and Harefield NHS Foundation Trust and Imperial College London. J. Jacob was supported by Wellcome Trust Clinical Research Career Development Fellowship 209553/Z/17/Z

\section{References}

1 Richeldi L, du Bois RM, Raghu G, et al. Efficacy and safety of nintedanib in idiopathic pulmonary fibrosis. $N$ Engl $J$ Med 2014; 370: 2071-2082.

2 King TE, Bradford WZ, Castro-Bernardini S, et al. A phase 3 trial of pirfenidone in patients with idiopathic pulmonary fibrosis. N Engl J Med 2014; 370: 2083-2092.

3 Wells AU. Forced vital capacity as a primary end point in idiopathic pulmonary fibrosis treatment trials: making a silk purse from a sow's ear. Thorax 2013; 68: 309-310.

4 Jacob J, Bartholmai BJ, Rajagopalan S, et al. Predicting outcome in idiopathic pulmonary fibrosis using automated CT analysis. Am J Respir Crit Care Med 2018; 198: 767-776.

5 Bates D, Mächler M, Bolker B, et al. Fitting linear mixed-effects models using lme4. J Stat Softw 2015; 67: 1-48.

6 Jacob J, Bartholmai B, Rajagopalan S, et al. Mortality prediction in idiopathic pulmonary fibrosis: evaluation of automated computer tomographic analysis with conventional severity measures. Eur Respir J 2017; 49: 1601011.

7 Jacob J, Bartholmai B, Rajagopalan S, et al. Automated quantitative CT versus visual CT scoring in idiopathic pulmonary fibrosis: validation against pulmonary function. J Thorac Imaging 2016; 31: 304-311.

8 IBM Corp. IBM SPSS Statistics for Windows VA, New York, IBM Corp., 2011.

9 Saketkoo LA, Mittoo S, Huscher D, et al. Connective tissue disease related interstitial lung diseases and idiopathic pulmonary fibrosis: provisional core sets of domains and instruments for use in clinical trials. Thorax 2014; 69: 436-444. 